\title{
Avaliação das Habilidades Cognitivo-Linguísticas de Aprendizagem em Crianças que Gaguejam
}

\section{Evaluation of Cognitive-Linguistic Learning Skills in Children Who Stutter}

\section{RESUMO}

Objetivo: Avaliar o desempenho nas habilidades cognitivo-linguísticas de aprendizagem em crianças com e sem gagueira em fase de alfabetização. Metodologia: Estudo de caso-controle, descritivo, transversal e quantitativo, realizado com uma amostra de 20 crianças, de ambos os sexos, em fase de alfabetização, que foram alocadas em dois grupos, na faixa etária de seis a doze anos. O grupo I: formado por dez criancas com gagueira do desenvolvimento persistente e o grupo II: composto por dez crianças sem gagueira. A coleta de dados foi realizada por meio do protocolo de Avaliação das habilidades cognitivo-linguísticas - versão coletiva e individual. A versão coletiva é composta pelas habilidades de escrita, aritmética, processamento auditivo e processamento visual; e a versão individual é composta pelas habilidades de leitura, metalinguística, processamento auditivo, processamento visual e velocidade de processamento. A análise dos dados foi realizada por meio do Teste t-Student, ao nível de significância de $5 \%$. Resultados: Os resultados deste estudo evidenciaram diferença significante, sugerindo maiores dificuldades do grupo que gagueja em relação ao grupo de crianças sem gagueira, nos subtestes de conhecimento do alfabeto $(p=0,008)$, aliteração $(p=0,004)$, rima $(p=0,002)$, segmentação silábica $(p=0,002)$, memória direta de dígitos $(p=0,004)$, repetição de palavras $(p=0,039)$, ritmo $(0,002)$ e memória indireta de dígitos $(p=0,002)$. Conclusão: Crianças que gaguejam possuem dificuldades de aprendizagem, relacionadas as habilidades metalinguísticas e de processamento auditivo.

\section{DESCRITORES}

Fonoaudiologia. Gagueira. Aprendizagem. Leitura. Alfabetização.

\begin{abstract}
Objective: To evaluate cognitive-linguistic learning skills in children with and without stuttering in the literacy phase. Methodology: Descriptive, cross-sectional, and quantitative case-control study. It was carried out with a sample of 20 children, of both sexes, in the literacy phase, who were allocated in two groups, in the age range of 6 to 12 years. Group I: composed of 10 children with stuttering of persistent development and group II: composed of 10 children without stuttering. Data collection was performed through the Cognitive-Linguistic Skills Assessment protocol - collective and individual version. The collective version consists of writing, arithmetic, auditory processing and visual processing skills; and the individual version is composed of reading, metalinguistic, auditory processing, visual processing and processing speed. Data were analyzed using the Student's $\mathrm{t}$-Test at a significance level of $5 \%$. Results: The results of this study showed a significant difference, suggesting greater difficulties of the group that stutters in relation to the group without stuttering, in alphabet knowledge subtests $(p=0.008)$, alliteration $(p=0.004)$, rhyme $(p=0.002)$, repetition of words $(p=0.039)$, rhythm $(p=0.002)$ and indirect memory of digits $(p=0.002)$. Conclusion: Children who stutter have learning difficulties in metalinguistics skills and auditory processing.
\end{abstract}

\section{DESCRIPTORS}

Speech. Language and Hearing Sciences. Stuttering. Learning. Reading. Literacy.

\footnotetext{
${ }^{1}$ Fonoaudióloga, Centro Universitário de João Pessoa - Unipê, João Pessoa (PB), Brasil.

${ }^{2}$ Fonoaudióloga, Doutora em Modelos de Decisão em Saúde, Professora do Centro Universitário de João Pessoa - Unipê, João Pessoa (PB), Brasil.

${ }^{3}$ Fonoaudióloga, Doutora em Linguística, Professora do Departamento de Fonoaudiologia e do Programa de Pós-Graduação em Linguística da Universidade Federal da Paraíba - UFPB, João Pessoa (PB), Brasil.

${ }^{4}$ Fonoaudiólogo, Doutor em Linguística, Professor do Centro Universitário de João Pessoa - Unipê, João Pessoa (PB), Brasil.
} 
$\mathrm{A}$ fluência é uma habilidade da comunicação, relacionada ao aspecto de produção de fala, de continuidade, velocidade e/ou esforço nos quais os níveis da linguagem, semânticos, fonológicos, morfológicos e/ou sintáticos são produzidos ${ }^{1}$.

A gagueira é o mais incidente e prevalente dos distúrbios da fluência ${ }^{2-4}$. As principais características associadas e diagnósticas da gagueira são o aparecimento de disfluências involuntárias na fala e a alteração no tempo da fala ${ }^{5-7}$. As disfluências evidenciam-se pela presença de repetições frequentes ou prolongamentos de sons ou sílabas, bloqueios e por outros tipos de disfluências da fala ${ }^{8}$, incluindo palavras incompletas, repetições de palavras monossilábicas e palavras produzidas com excesso de tensão física.

No Brasil, existe uma incidência de $5 \%$ de gagueira, o que corresponde a cerca de 10 milhões de brasileiros que nesse momento estão passando por um período de gagueira. Sendo a prevalência na população de $1 \%$, no que concerne a 2 milhões de pessoas que gaguejam de forma crônica ${ }^{9}$. Isso remete ao pensamento do quanto a gagueira é comum na população brasileira e o quanto estes indivíduos, principalmente as crianças, carecem de um olhar voltado para estas, devido a sua fragilidade e exposição no meio social, principalmente no ambiente escolar.

Observa-se que os sintomas iniciais da gagueira, normalmente, surgem na faixa etária de dois a cinco anos de idade, acometendo principalmente os meninos e indivíduos com histórico familiar e de atraso de fala e de linguagem positivo, na maioria dos $\operatorname{casos}^{10,11}$. Podem-se encontrar, ainda, movimentos motores associados, como: movimentos involun- tários de cabeça, batimentos de asa de nariz, língua, piscar de olhos, movimentos respiratórios de membros superiores e de membros inferiores ${ }^{6,10}$. Além disso, outra característica que alguns estudos descrevem é que crianças que gaguejam podem apresentar dificuldades de aprendizagem ${ }^{12}$.

Para que a criança passe pelo processo de escolarização, uma série de habilidades cognitivo-linguísticas, necessariamente, deverão ser previamente adquiridas ou desenvolvidas, que consistem de: leitura, escrita, habilidade metalinguística, processamento auditivo, processamento visual, velocidade de processamento e raciocínio lógico ${ }^{13}$.

É descrito na literatura que crianças com gagueira podem apresentar dificuldades de aprendizagem ${ }^{12}$, que pode, dessa forma, ser resultado ou provocar dificuldades nas habilidades cognitivo-linguísticas. Desse modo, questiona-se: crianças que gaguejam apresentam desempenho nas habilidades cognitivo-linguísticas diferente das que não gaguejam? Existe relação da gagueira com as habilidades cognitivas e linguísticas no processo de aprendizagem?

Esses questionamentos surgiram devido ao fato da atenção dos profissionais de saúde e da educação estar, muitas vezes, voltada apenas à fala das crianças que gaguejam, sendo importante considerar que outras habilidades do neurodesenvolvimento podem estar funcionando de forma diferenciada na população.

Sendo assim, o presente estudo teve por objetivo avaliar o desempenho nas habilidades cognitivo-linguísticas de aprendizagem em crianças com e sem gagueira em fase de alfabetização. 


\section{METODOLOGIA}

Trata-se de um estudo de caso-controle, descritivo, transversal e quantitativo, tendo sido submetido e aprovado pelo Comitê de Ética em Pesquisa da instituição de origem sob o número CAEE 02771018.2.0000.5176. O trabalho foi desenvolvido nas clínicas de Fonoaudiologia de duas instituições de ensino superior e em uma escola pública de ensino fundamental.

\section{Amostra}

O estudo foi realizado com uma amostra por conveniência, que contou com a participação de 20 crianças, sendo duas (20\%) do sexo feminino e 18 ( $80 \%$ ) do sexo masculino, na faixa etária de seis a 12 anos, em fase de alfabetização que cursavam do $1^{\circ}$ ano ao $8^{\circ}$ do ensino fundamental, que foram pareados por sexo, idade e escolaridade e alocados em dois grupos a partir dos seguintes critérios de elegibilidade:

- Grupo com gagueira (GI): formado por dez crianças com diagnóstico de gagueira do desenvolvimento persistente (GDP); com no mínimo 3\% de disfluências típicas da gagueira; que na ocasião do estudo, estavam ou não em terapia fonoaudiológica para a gagueira; advindos do serviço de fonoterapia e de triagem das duas instituições de ensino superior.

- Grupo sem gagueira (GII): composto por dez crianças sem gagueira; sem queixas atual ou pregressa de gagueira; com menos de $3 \%$ de disfluências típicas da gagueira; que frequentam uma escola pública ensino fundamental.

Como critério de exclusão para ambos os grupos foram consideradas: a falta de interesse em participar da pesquisa; não concluir a avaliação; crianças apresentarem alteração linguística, neurológica ou auditiva que comprometesse a realização da pesquisa, aspectos averiguados através da história clínica infantil e triagens específicas; e os pais e as crianças não assinarem respectivamente o Termo de consentimento livre e esclarecido e o Termo de assentimento.

\section{Procedimentos e Instrumentos}

Todos os participantes, foram submetidos à uma anamnese, que coletou as seguintes informações: desenvolvimento global infantil, desenvolvimento da linguagem, aspectos da aprendizagem, histórico médico geral e tratamentos prévios a pesquisa. Além disso, os responsáveis dos participantes do GI responderam as informações acerca do surgimento das disfluências, evolução do quadro clínico da gagueira, fatores qualitativos associados e tratamento anterior e prévio.

Em seguida, todos os participantes passaram por uma avaliação fonoaudiológica, por meio do protocolo de avaliação da fluência da fala ${ }^{14}$. Para este protocolo, o material de fala coletado de cada criança foi a fala espontânea (média de 200 sílabas fluentes), por meio da contagem de um filme, esse material foi gravado por meio de áudio.

As gravações foram transcritas e, em seguida, foi realizado o mapeamento das outras disfluências (OD) e disfluências típicas 
da gagueira (DTG), realização dos cálculos de frequência de rupturas - $\%$ de DTG e a descontinuidade de fala (DF) - e de velocidade de fala - palavras por minuto (PPM) e sílabas por minuto (SPM). As tipologias de OD foram: hesitação, interjeição, revisões, repetição de parte do enunciado, repetição de frases, repetição de palavras não monossilábicas e palavras incompletas. As tipologias que fazem parte das DTG foram: repetição de palavras monossilábicas, repetição de parte da palavra, repetição de sons, pausas, bloqueios, prolongamentos e intrusões ${ }^{15}$.

Por fim, os participantes passaram pelo processo de avaliação da aprendizagem, por meio do Protocolo de habilidades cognitivo-linguísticas (PAHCL), desenvolvido por Capellini, Smythe e Silva ${ }^{13}$.

O PAHCL possui o objetivo de investigar os aspectos da aprendizagem da criança, como: a leitura, a escrita, a habilidade metalinguística, o raciocínio lógico, o processamento auditivo, o processamento visual e a velocidade de processamento.

O protocolo é composto por duas versões: a coletiva e a individual, ambas as versões foram aplicadas de forma individual em cada criança. A versão coletiva avalia as habilidades de escrita, aritmética, processamento auditivo e processamento visual, sendo composta por cinco subtestes: 1) conhecimento do alfabeto, 2) cópia de formas, 3) cálculo matemático, 4) ditado de palavras e não palavras e 5) memória direta de dígitos.

A versão individual avalia as habilidades de leitura, metalinguística, processamento auditivo, processamento visual e velocidade de processamento, sendo composta por 13 subtestes: 1) leitura de palavras, 2) leitura de não palavras, 3) aliteração, 4) rima, 5) repetição de palavras, 6) repetição de não palavras, 7) ritmo, 8) segmentação silábica, 9) nomeação rápida de figuras, 10) nomeação rápida de dígitos, 11) memória visual para figuras, 12) discriminação de sons e 13) memória indireta de dígitos.

Foram analisados os escores apresentados pelas crianças em cada prova e comparados com os parâmetros de normalidade apresentados no protocolo de acordo com a série $^{13}$. Ressalta-se que todos os procedimentos foram realizados em uma única sessão com duração de 2 horas.

\section{Análise dos dados}

Os dados foram transferidos para uma planilha eletrônica digital para realização de testes estatísticos pelo software Statistical Package for Social Sciences (SPSS), versão 20.0. Foi realizada estatística descritiva, com realização da média e do desvio-padrão das variáveis e inferencial. Para comparação das médias dos aspectos da fluência e dos aspectos de habilidades cognitivo-linguísticas foi utilizado o Teste $t$-Student. Em todas as análises estatísticas, foi adotado o nível de significância de $5 \%$.

\section{RESULTADOS}

$\mathrm{Na}$ comparação das médias entre os grupos, foram observadas diferenças estatísticas entre as crianças que gaguejavam e as que não gaguejavam. O grupo com gagueira apresentou uma maior quantidade de DTG $(p=0,001)$, na $\%$ DTG $(p=0,001)$ e $\%$ DF $(p=0,002)$ e menores valores de PPM $(p=0,010)$ e SPM $(p=0,015)$ (Tabela 1). 
Tabela 1. Comparação de médias nos escores dos parâmetros da fluência

\begin{tabular}{lccccc}
\hline \multirow{2}{*}{ VARIÁVEL } & \multicolumn{2}{c}{ GAGUEIRA } & \multicolumn{2}{c}{ SEM GAGUEIRA } & \multirow{2}{*}{ p-valor } \\
\cline { 2 - 5 } & Média & DP & Média & DP & 0,067 \\
Quantidade de OD & 9 & 6,89 & 4,5 & 2,41 & $<0,001^{*}$ \\
Quantidade de DTG & 11,8 & 5,73 & 1 & 0,81 & $<0,001^{*}$ \\
\% DTG & 5,86 & 2,87 & 0,49 & 0,39 & $0,002^{*}$ \\
\% DF & 10,16 & 6,30 & 2,74 & 1,35 & $0,010^{*}$ \\
PPM & 71,04 & 17,86 & 94,07 & 18,07 & $0,015^{*}$ \\
SPM & 118,64 & 29,32 & 157,74 & 35,65 & 0,74 \\
\hline
\end{tabular}

Legenda: $\mathrm{OD}=$ outras disfluências; $\mathrm{DTG}=$ disfluências típicas da gagueira; \%DTG: porcentagem de disfluências típicas da gagueira; \%DF= porcentagem de descontinuidade de fala; $P P M=$ palavras por minuto; $S P M=$ sílabas por minuto; $\mathrm{DP}=$ desvio-padrão. * Valores estatisticamente significantes $(p \leq 0,05)$ - Teste t-Student

Tabela 2. Comparação de médias nos escores dos parâmetros das habilidades cognitivolinguísticas

\begin{tabular}{|c|c|c|c|c|c|c|}
\hline \multirow{2}{*}{ HABILIDADES } & \multirow{2}{*}{ SUBTESTES } & \multicolumn{2}{|c|}{ GAGUEIRA } & \multicolumn{2}{|c|}{ SEM GAGUEIRA } & \multirow{2}{*}{ p-valor } \\
\hline & & Média & DP & Média & DP & \\
\hline \multirow{3}{*}{ Leitura } & $\begin{array}{l}\text { Conhecimento } \\
\text { Alfabético }\end{array}$ & 17,20 & 10,60 & 18 & 9,36 & 0,860 \\
\hline & Leitura de palavras & 32,800 & 29,84 & 20,60 & 28,39 & 0,361 \\
\hline & $\begin{array}{l}\text { Leitura de não } \\
\text { palavras }\end{array}$ & 6,10 & 4,33 & 5,30 & 4,71 & 0,697 \\
\hline \multirow[b]{2}{*}{ Escrita } & Ditado de palavras & 13,50 & 11,05 & 9,90 & 11,58 & 0,486 \\
\hline & $\begin{array}{c}\text { Ditado de não } \\
\text { palavras }\end{array}$ & 3,30 & 3,02 & 3,20 & 3,67 & 0,948 \\
\hline \multirow{3}{*}{ Metalinguística } & Aliteração & 6,20 & 3,01 & 8 & 2 & 0,133 \\
\hline & Rima & 10,50 & 5,31 & 15,40 & 4,76 & $0,044^{*}$ \\
\hline & Segmentação silábica & 15,20 & 4,80 & 19,20 & 1,47 & $0,022^{*}$ \\
\hline Raciocínio Lógico & Cálculo Matemático & 5,40 & 6,25 & 7,60 & 7,77 & 0,495 \\
\hline
\end{tabular}

Legenda: DP= desvio-padrão. * Valores estatisticamente significantes $(p \leq 0,05)-$ Teste t-Student

Em relação às habilidades cognitivo-linguísticas, ocorreu diferença estatística na comparação das médias dos grupos nos subtestes: rima $(p=0,044)$ e segmentação silábica $(p=0,022)$ (Tabela 2).

$\mathrm{Na}$ análise dos grupos, verificou-se diferença estatística na habilidade de processamento auditivo no que se refere à repetição de não palavras $(p=0,005)$, ritmo $(p=0,003)$, discriminação de sons $(p=0,031)$ e memória indireta de dígitos $(p=0,04)$ (Tabela 3$)$.
No que se refere às habilidades cognitivo-linguísticas de processamento visual, existiu diferença estatística apenas no subteste de memória visual com figuras $(p=0,003)$, na comparação das médias dos grupos (Tabela 3).

\section{DISCUSSÃO}

Nesta pesquisa, foi possível observar diferença estatística na média nas medidas 
Tabela 3. Comparação de médias nos escores dos parâmetros das habilidades cognitivolinguísticas

\begin{tabular}{|c|c|c|c|c|c|c|}
\hline \multirow[t]{2}{*}{ HABILIDADES } & \multirow[t]{2}{*}{ SUBTESTES } & \multicolumn{2}{|c|}{ GAGUEIRA } & \multicolumn{2}{|c|}{$\begin{array}{c}\text { SEM } \\
\text { GAGUEIRA }\end{array}$} & \multirow[t]{2}{*}{ p-valor } \\
\hline & & Média & DP & Média & DP & \\
\hline \multirow{6}{*}{ Processamento Auditivo } & Memória direta de dígitos & 5,30 & 2,66 & 6,90 & 3,07 & 0,230 \\
\hline & Repetição de palavras & 4,10 & 1,72 & 5,10 & 1,37 & 0,169 \\
\hline & $\begin{array}{l}\text { Repetição de não } \\
\text { palavras }\end{array}$ & 4,10 & 1,59 & 6 & 0,94 & $0,005^{*}$ \\
\hline & Ritmo & 1,90 & 1,10 & 4,40 & 2,06 & $0,003^{*}$ \\
\hline & Discriminação de sons & 18,80 & 1,61 & 20 & 0 & $0,031^{*}$ \\
\hline & $\begin{array}{c}\text { Memória indireta de } \\
\text { dígitos }\end{array}$ & 2,80 & 1,39 & 4,20 & 1,54 & $0,04^{*}$ \\
\hline \multirow{2}{*}{ Processamento visual } & Cópia de Formas & 3,30 & 0,67 & 3,50 & 0,70 & 0,526 \\
\hline & $\begin{array}{c}\text { Memória visual com } \\
\text { figuras }\end{array}$ & 3 & 1,33 & 4,90 & 1,10 & $0,003^{*}$ \\
\hline \multirow{2}{*}{$\begin{array}{l}\text { Velocidade de } \\
\text { processamento }\end{array}$} & $\begin{array}{l}\text { Nomeação rápida de } \\
\text { figuras }\end{array}$ & 41,70 & 12,22 & 43,50 & 11,59 & 0,739 \\
\hline & $\begin{array}{c}\text { Nomeação rápida de } \\
\text { dígitos }\end{array}$ & 44,70 & 15,89 & 42,10 & 14,20 & 0,704 \\
\hline
\end{tabular}

Legenda: DP= desvio-padrão. * Valores estatisticamente significantes $(p \leq 0,05)-$ Teste $t-S t u d e n t$

de frequência de rupturas entre os grupos (Tabela 1), como a \% de DTG, indicando que todas as crianças do estudo que gaguejam atenderam ao critério para o diagnóstico de gagueira, ou seja, o indivíduo apresentar, no mínimo, 3\% de DTG na fala espontânea ${ }^{16}$. Esses resultados corroboram com as evidências ${ }^{2}$ que as crianças que gaguejam apresentam uma maior quantidade no total de \%DTG e \%DF na fala espontânea, quando comparado a crianças que não gaguejam.

Em relação à velocidade de fala das crianças que gaguejam, os resultados calculados pela quantidade de itens falados por minuto, quanto ao fluxo de palavras por minuto (PPM) e de sílabas por minuto (SPM), foram menores e dessa forma esperados (Tabela 1), uma vez que na fala os indivíduos que gaguejam apresentam dificuldade na velocidade articulatória, que diminui a taxa de informação, em comparação às crianças que não gaguejam ${ }^{16,17}$.

Na comparação de média da habilidade de leitura e escrita, os resultados não indicaram diferença estatística entre os grupos nos subtestes de conhecimento do alfabeto, leitura de palavras e não palavras, ditado de palavras e de não palavras (Tabela 2).

No que se refere à habilidade metalinguística, ocorreu diferença estatística na comparação de média entre os grupos nos subtestes de rima e segmentação silábica (Tabela 2).

Estes resultados sugerem que o grupo de crianças com gagueira apresentam mais dificuldades na capacidade de pensar na própria 
língua, ou seja, no que se refere a estrutura sintática, semântica e fonológica. O prejuízo na habilidade metalinguística pode refletir neste grupo em dificuldades para manipular/ segmentar e operar as palavras, sílabas e fonemas ${ }^{18-21}$.

As habilidades metalinguísticas contribuem para o processo da alfabetização e da aprendizagem da leitura e escrita, pois facilitam o princípio da correspondência grafema-fonema ${ }^{13}$.

Em avaliações da consciência fonológica de crianças com e sem gagueira verificou-se que as que gaguejam apresentaram desempenho significativamente diferente na codificação fonológica, porém de forma sutil, em comparação aos seus pares tipicamente fluentes ${ }^{20}$. Tal pesquisa corroborou com os resultados deste estudo que demonstrou que as crianças que gaguejam tiveram nos três subtestes da habilidade metalinguística desempenho pior em comparação às que não gaguejam (Tabela 2).

Em um outro estudo, pode-se avaliar a segmentação e a rima de crianças com e sem gagueira, na faixa etária de sete a treze anos, foi observado que o grupo que gagueja não apresentou alteração em nível de segmentação e rima em comparação com as crianças sem gagueira, porém, foi possível evidenciar no estudo que existiram dificuldades de segmentação com o aumento da complexidade fonológica dos estímulos ${ }^{18}$.

Em relação à habilidade de processamento auditivo, os resultados indicaram diferença estatística na média entre os grupos nos subtestes de repetição de não palavras, ritmo, de discriminação de sons e da memória indireta de dígitos (Tabela 3). De acordo com estes resultados, observou-se que as crianças com gagueira podem apresentar dificuldades no processamento auditivo, mas especificamente nas habilidades auditivas que envolvam a detecção, a discriminação, a compreensão, o reconhecimento e a memória auditiva ${ }^{13,22}$

Os subtestes do presente estudo quanto ao processamento auditivo envolvem além da informação auditiva o acesso à memória ${ }^{13}$, pois os subtestes de memória direta e indireta de dígitos e de repetição de números e de palavras necessitam do acesso ao léxico e de um bom armazenamento da informação para a reprodução do que lhe foi solicitado. As dificuldades observadas podem ser indicativas que as crianças que gaguejam podem apresentar dificuldades de memória.

Indivíduos que gaguejam apresentam como característica o comprometimento do ritmo, podendo estar associado ao transtorno do processamento auditivo ${ }^{23-27} \mathrm{e}$, no presente estudo, as crianças do grupo que gaguejam apresentaram dificuldades no ritmo.

A habilidade de processamento auditivo reflete a efetividade com que o sistema nervoso central (SNC) utiliza a informação auditiva, ou seja, interpreta e/ou codifica os estímulos sonoros ${ }^{24}$. Estudo ${ }^{25}$ que caracterizou e comparou o desempenho das crianças com e sem gagueira nos testes de processamento auditivo, constatou que crianças com gagueira apresentaram alteração no processamento auditivo em relação a crianças sem gagueira.

$\mathrm{Na}$ habilidade de processamento visual, na comparação da média, com relação aos subtestes de cópia de formas e memória visual de figuras, apenas, este último apresentou diferença significante (Tabela 
3). Isto pode ser explicado devido aos possíveis comprometimentos nos componentes da habilidade de processamento visual dos participantes, que envolvem a memória de trabalho, a organização espacial e o processamento da informação visual ${ }^{28}$.

Mediante à avaliação das habilidades cognitivo-linguísticas, foi notório observar que as maiores dificuldades do grupo de crianças com gagueira estiveram relacionadas às habilidades metalinguísticas e de processamento auditivo. Tais habilidades devem ser contempladas no processo terapêutico pelo fonoaudiólogo e, no ambiente escolar, pelos professores, a fim de que possam utilizar de estratégias eficazes a fim de promover um melhor processo de aprendizagem para as crianças que gaguejam, tendo em vista, a dificuldade das mesmas encontradas nesse estudo.

A parceria entre professores e fonoaudiólogos deve promover uma boa evolução no processo de ensino-aprendizagem da criança que gagueja, identificando estratégias, metodologias e atividades necessárias para concretizar a inclusão da criança com gagueira na sala de aula, a fim de promover uma aprendizagem significativa, permitindo, enquanto aluno, superar os desafios postos e evitar possíveis frustrações futuras.
Nesse contexto, a atuação do fonoaudiólogo poderá auxiliar as crianças que gaguejam a superar rendimentos escolares abaixo do esperado, além do enfoque a fluência da fala e contribuir para uma melhora nessas habilidades cognitivas e linguísticas que, consequentemente, refletirá de forma positiva no processo de ensino-aprendizagem dessas crianças.

\section{CONCLUSÃO}

Crianças com gagueira apresentaram dificuldades nas habilidades cognitivo-linguísticas, com um pior desempenho nas habilidades metalinguísticas e de processamento auditivo.

Dessa forma, é necessário que este grupo passe por uma avaliação específica e mais aprofundada de cada habilidade dentro do ambiente terapêutico, tendo em vista que o protocolo utilizado é um instrumento de rastreio da aprendizagem, analisando apenas alguns domínios dentro de cada habilidade. É importante que o fonoaudiólogo e os professores desenvolvam estratégias em conjunto para auxiliar as crianças com gagueira a superar essas possíveis dificuldades de aprendizagem. 


\section{REFERÊNCIAS}

1. American Speech-Language-Hearing Association (ASHA). Terminology pertaining to fluency and fluency disorders: Guidelines. ASHA Special Interest Division 4: Fluency and Fluency Disorder. ASHA (Suppl. 19). 1999; 41:29-36.

2. Fiorin M, Ugarte CV, Capellini AS, Oliveira CMC. Fluência da leitura e da fala espontânea de escolares: estudo comparativo entre gagos e não gagos. Rev. CEFAC. 2015;17(1):151-8.

3. Whitfield JA, Delong C, Goberman AM, Blomgren M. Fluency adaptation in speakers with Parkinson disease: a motor learning perspective. International Journal of Speech-Language Pathology 2017;30:1-9.

4. Souza MCL. Perfil da Fluência de fala de escolares segundo a gravidade da gagueira [dissertação]. Faculdade de Filosofia e Ciências - UNESP; Marília; 2018.

5. Maguire G. Franklin D, Vatakis NG, Morgenshtern E, Denko T, Yaruss JS, et a. Exploratory randomized clinical study of pagoclone in persistent developmental stuttering: the examining pagoclone for persistent developmental stuttering study. Journal of Clinical Psychopharmacology. 2010; 30(1):48-56.

6. American Psychiatric Association (APA). Manual Diagnóstico e Estatístico de Transtornos Mentais - DSM 5. Tradução de Maria Inês Correa Nascimento et al; revisão técnica Aristides Volpato Cordiolo. 5. ed. Porto Alegre: Artes Médicas, 2014.

7. Ambrose NG, Yairi E, Loucks TM, Seery CH, Thronoburg $\mathrm{R}$. Relation of motor, linguistic and temperament factors in epidemiologic subtypes of persistent and recovered stuttering: Initial findings. Journal of Fluency Disorders. 2015; 45:12-26.

8. Ritto AP, Juste FS, Andrade CRF. Impacto do uso do SpeechEasy® nos parâmetros acústicos e motores da fala de indivíduos com gagueira. Audiology Communication Research. 2015;20(1):1-9.

9. Instituto Brasileiro de Fluência (IBF). 2018.

10. Duarte TF, Crenitte PAP, Lopes-Herrera AS. Caracterização dos indivíduos com distúrbios da fluência, atendidos na clínica-escola do curso de fonoaudiologia da USP-Bauru. Rev. CEFAC, São Paulo. 2009; 11(3):396-405.

11. Maciel TM, Celeste LC, Martins-Reis VO. Gagueira infantil: subsídios para pediatras e profissionais de saúde. Rev Med Minas Gerais 2013; 23(3):350-6.
12. Briley PM, Junior CE. The Coexistence Of Disabling Conditions In Children Who Stutter: Evidence From The National Health Interview Survey. 2018.

13. Capellini AS, Smythe I, Silva C. Protocolo de Avaliação de Habilidades Cognitivo-Linguísticas. Livro do Profissional e do Professor. $1^{\text {a }}$ ed (revisada, e atualizada). Marília: Fundepe, 2012.

14. Andrade, C.R.F. Fluência. In: Andrade, C.R.F.; et al. ABFW: teste de linguagem infantil nas áreas de fonologia, vocabulário, fluência e pragmática. $2^{\mathrm{a}}$ ed (revisada, ampliada e atualizada). Barueri: Pró-Fono; 2004. p. 51-82.

15. Oliveira CMC, Correia DV, Ninno CQMS. Avaliação da fluência. In: Lamônica DAC, Britto DBO. Tratado de Linguagem: perspectivas contemporâneas. Ribeirão Preto: Booktoy; 2017. p. 107-13.

16. Pinto JCBR, Schiefer AM, Ávila CRB. Disfluências e velocidade de fala em produção espontânea e em leitura oral em indivíduos gagos e não gagos. Audiology Communication Research. 2013;18(2):63-70.

17. Arcuri CF, Ishii C, Schiefer AM, Pereira LD. Fatores de risco auditivo em indivíduos gagos. Fono Atual. 2004; 28(7):4-10.

18. Sasisekaran J, Byrd CT. A preliminary investigation of segmentation and rhyme abilities of children who stutter. Journal of Fluency Disorders. 2013; 38(2):222-34. .

19. Silva C, Capellini S.A. Desempenho de escolares com e sem transtorno de aprendizagem em leitura, escrita, consciência fonológica, velocidade de processamento e memória de trabalho fonológica. Rev. psico pedag. 2013; 30(91):3-11.

20. Pelczarski MK, Yaruss JS. Phonological encoding of young children who stutter. Journal of Fluency Disorders. 2014; 39:12-24.

21. Gonçalves TS. Habilidade metalinguística pragmática em crianças em idade escolar. Revista Digital do Programa de Pós-Graduação em Letras da PUCRS. Porto Alegre. 2016; 9:114-25.

22. Bastos FN, Fleig R, Nascimento IB. Análise das habilidades auditivas em uma criança deficiente auditiva oralizada e portadora de hiv: estudo de caso. Rev. CEFAC 2010;12(4):700-8.

23. Andrade CRF. Abordagem neurolingüística e motora da gagueira. In: Ferreira LP, Befi-Lopes DM, Limongi SCO. Tratado de Fonoaudiologia. São Paulo: Roca; 2004:1001-16 
24. Andrade AN, Gil D, Schiefer AM, Pereira LD. Processamento auditivo em gagos: análise do desempenho das orelhas direita e esquerda. Revista da sociedade brasileira de Fonoaudiologia. São Paulo. 2008;13(1):20-9.

25. Silva R, Oliveira CMC, Cardoso ACV. Aplicação dos testes de padrão temporal em crianças com gagueira desenvolvimental persistente. Rev. CEFAC. 2011;13(5):902-908.

26. Carvalho NG, Novelli CV, Colella-Santos MF. Fatores na infância e adolescência que podem influenciar o processamento auditivo: revisão sistemática. Rev. CEFAC. 2015;17(5):1590-603.

27. Prestes R, Andrade AN, Santos RBF, Marangoni AT, Shiefer AM, Gil D.Temporal processing and long-latency auditory evoked potential in stutterers. Brazilian J Otorhinolaryngolog. 2017; 83(2):142-6.
28. Silva C, Capellini SA. Correlação entre habilidades cognitivo-linguísticas em escolares com dificuldades de aprendizagem. Rev. psicopedag., São Paulo. 2012;29(89):183-93.

\section{CORRESPONDÊNCIA}

Ivonaldo Leidson Barbosa Lima

Curso de Fonoaudiologia.

Centro Universitário de João Pessoa - UNIPÊ

BR 230 - Km 22, Água Fria -

CEP 58053-000 João Pessoa - PB.

E-mail: ivonaldo.lima@unipe.edu.br 